\title{
Exploration of potential mechanisms for parasite induced anorexia of sheep through modelling
}

\section{YCSM Laurenson $^{1}$, SC Bishop ${ }^{1}$, I Kyriazakis ${ }^{2,3}$}

${ }^{1}$ The Roslin Institute and R(D)SVS, University of Edinburgh, Edinburgh, Midlothian, United Kingdom, ${ }^{2}$ University of Thessaly, Kardhitsa, Greece, ${ }^{3}$ Newcastle University, Newcastle upon Tyne, United Kingdom

Email:yan.laurenson@roslin.ed.ac.uk

Introduction Gastrointestinal (GI) parasitism results in a reduction of the voluntary food intake, and hence productivity, of sheep. Previously an in silico simulation model of Teladorsagia circumcincta infections was developed (Vagenas et al. 2007), describing nutrient utilisation, host-parasite interactions and the development of immunity, for the purpose of predicting effects of host nutrition and genotype on the progression of GI infections. However, a difficult feature of such models is appropriately modelling parasite-induced anorexia. Although the precise causes of anorexia are unknown, two mechanisms for modelling anorexia can be invoked; a reduction in intrinsic growth rate (IGR) and a reduction in food intake (FIR), both as a function of parasite burden. This paper explores these mechanisms, and their consequences on predicted live weight (LW), food intake (FI) and faecal egg count (FEC).

Materials and methods For both mechanisms the model of Vagenas et al. (2007) was used to simulate an immunologically naïve lamb from 2 to 6 months of age. The lamb modelled was similar to a Scottish Blackface, with an initial and mature live weight of $18 \mathrm{~kg}$ and $85 \mathrm{~kg}$, respectively. The lamb was offered, in silico, one of two qualities of grass ad libitum; viz. good quality (12.6MJ/kg of dry matter (DM) metabolizable energy (ME) and $190 \mathrm{~g} / \mathrm{kg}$ DM crude protein (CP)) and poor quality (7.5MJ ME $/ \mathrm{kg} \mathrm{DM}$ and $97 \mathrm{~g} \mathrm{CP} / \mathrm{kg} \mathrm{DM})$, respectively. The good quality grass is presumed to be of sufficient quality to be non-limiting (AFRC, 1993). The lamb was challenged in silico with either control, 1000 or 5000 infective larvae per day, from day one, corresponding to a range of challenge levels that normally lead to subclinical $T$. circumcinta infections (Coop et al. 1985). Predictions of FI, LW and FEC were assessed over time, with detailed comparisons presented here at day 60 , a time point when impacts of anorexia are clearly observed across all scenarios investigated in this study.

Results Predicted trait values, assessed 60 days post infection, are summarised in Table 1, with anorexia mechanisms, grass quality and larval challenge all affecting predicted performance. For the good quality grass there was little difference in the FEC between the two mechanisms, however, there were notable differences in predicted FI and LW. With the FIR mechanism, reductions were predicted for LW and FI, compared to the control, which increased with higher larval challenges, whereas with the IGR mechanism similar reductions for both larval doses were predicted. For the poor quality grass, with the IGR mechanism FI is determined by the bulkiness of the food and as a consequence predicted FI was not reduced below the maximum gut fill; i.e. pathogen challenge does not further reduce the already constrained resources and hence little impact of parasitism on poor quality grass was observed for IGR mechanism. With the FIR mechanism, predicted FI displayed a reduction greater than that imposed by the maximum gut fill, resulting in a large reduction in predicted LW and an increase in FEC. Therefore, the model containing the FIR mechanism predicted a severe effect of parasitism in lambs offered poor quality grass. Fig 1 shows the progression of the impact of larval dose and grass quality on LW for the FIR mechanism over time, and the large temporal impacts on poor quality grass can be seen.

Table 1 Model predictions 60 days post infection, for actual FEC, FI and LW as a proportion of the control lamb

\begin{tabular}{llllllll}
\hline \hline \multirow{2}{*}{$\begin{array}{l}\text { Grass } \\
\text { qual. }\end{array}$} & $\begin{array}{l}\text { Larval } \\
\text { dose }^{\dagger}\end{array}$ & \multicolumn{3}{l}{ IGR Mechanism } & \multicolumn{3}{c}{ FIR Mechanism } \\
\cline { 3 - 8 } Good & 1000 & 0.82 & 130 & 0.89 & 0.89 & 120 & 0.96 \\
& 5000 & 0.81 & 237 & 0.89 & 0.78 & 255 & 0.88 \\
\hline Poor & 1000 & 0.95 & 123 & 0.94 & 0.64 & 197 & 0.80 \\
& 5000 & 0.94 & 241 & 0.94 & 0.58 & 561 & 0.72 \\
\hline \hline
\end{tabular}

$\bar{\dagger}{ }^{\dagger}$ units $=$ larvae/day, ${ }^{\dagger}$ all values for FI (kg), FEC (eggs/g), and LW $(\mathrm{kg})$ are expressed as a proportion of the control lamb.

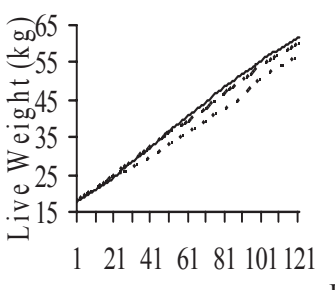

a. Good quality grass

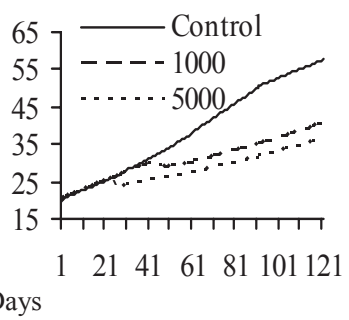

b. Poor quality grass

Figure $1 \mathrm{LW}$ for different challenge levels, FIR mechanism

Conclusions The impacts of the different anorexia mechanisms differ with both larval challenge rate and food quality, leading to different predicted impacts of parasitism. However, predictions of the impact of parasitism on LW, across different challenge levels, using the FIR mechanism are very similar to the experimental results reported by Coop et al. (1985) for good quality grass. These predictions impact on our understanding of the nature and implications of anorexia, and provide testable hypotheses for experimental verification.

Acknowledgements We thank BBSRC, Merial and the Bioscience KTP (formerly Genesis Faraday) for funding.

\section{References}

Coop, R.L., Graham, R.B., Jackson, F., Wright, S.E., Angus, K.W. 1985. Research in Veterinary Science. 38, 282-287.

Vagenas, D., Bishop, S.C., Kyriazakis, I. 2007. Parasitology. 134, 1263-1277.

Agricultural and Food Research Council. 1993. Energy and protein requirements of ruminants. An advisory manual prepared by AFRC Technical committee on responses to nutrients. CAB International, Wallingford, UK. 